\title{
Identification and validation of mRNA 3 'untranslated regions of DNMT3B and TET3 as novel competing endogenous RNAs of the tumor suppressor PTEN
}

\author{
KENNETH ANTHONY R. ROQUID ${ }^{1,2}$, KRIZELLE MAE M. ALCANTARA ${ }^{1,3}$ and REYNALDO L. GARCIA ${ }^{1}$ \\ ${ }^{1}$ Disease Molecular Biology and Epigenetics Laboratory, National Institute of Molecular Biology and Biotechnology, \\ National Science Complex, University of the Philippines Diliman, Quezon City, Metro Manila 1101, Philippines; \\ ${ }^{2}$ Department of Pharmacology, Max Planck Institute for Heart and Lung Research, D-61231 Bad Nauheim, Hesse, Germany
}

Received May 11, 2019; Accepted November 20, 2019

DOI: $10.3892 /$ ijo.2019.4947

\begin{abstract}
PTEN inactivation is a frequent event in oncogenesis. Multiple regulatory mechanisms such as promoter hypermethylation, antisense regulation, histone modifications, targeting by microRNAs (miRNAs/miRs) and regulation by transcription factors have all been shown to affect the tumor suppressor functions of PTEN. More recently, the functional involvement of competing endogenous RNAs (ceRNAs) in miRNA-dependent and coding-independent regulation of genes shed light on the highly nuanced control of PTEN expression. The present study has identified and validated DNA methyltransferase $3 \beta$ (DNMT3B) and TET methylcytosine dioxygenase 3 (TET3) as novel ceRNAs of PTEN, with which they share multiple miRNAs, in HCT116 colorectal cancer cells. miR-4465 was identified and characterized as a miRNA that directly targets and regulates all 3 transcripts via their 3'untranslated regions (3'UTRs) through a combination of luciferase reporter assays, abrogation of miRNA response elements (MREs) via site-directed mutagenesis, target protection of MREs with locked nucleic acids, RT-qPCR assays and western blot analysis. Competitive miRNA sequestration was demonstrated upon reciprocal 3'UTR overexpression and siRNA-mediated knockdown of their respective transcripts. Overexpression of DNMT3B or TET3 3'UTR promoted apoptosis and decreased migratory capacity, potentially because of shared miRNA sequestration and subsequent activation
\end{abstract}

Correspondence to: Professor Reynaldo L. Garcia, Disease Molecular Biology and Epigenetics Laboratory, National Institute of Molecular Biology and Biotechnology, National Science Complex, University of the Philippines Diliman, Ma. Regidor Street, Quezon City, Metro Manila 1101, Philippines

E-mail: reygarcia@mbb.upd.edu.ph

${ }^{3}$ Present address: Center for Gene Therapy, Abigail Wexner Research Institute, Nationwide Children's Hospital, 700 Children's Drive, Columbus, $\mathrm{OH} 43205$, USA

Key words: PTEN, TET methylcytosine dioxygenase 3, DNA methyltransferase $3 \beta$, competing endogenous RNA, microRNA-4465 of PTEN expression. Knockdown of TET3 and DNMT3B decoupled their protein-coding from miRNA-dependent, coding-independent functions. Furthermore, the findings suggested that the phenotypic outcome of ceRNAs is dictated largely by the number of shared miRNAs, and predictably, by the existence of other ceRNA networks in which they participate. Taken together, the findings of the present study identified DNMT3B and TET3 as novel ceRNAs of PTEN that may impact its dose-sensitive tumor suppressive function.

\section{Introduction}

The PTEN gene is a tumor suppressor that negatively regulates the phosphoinositol-3-kinase/AKT survival pathway (1). PTEN is important in sustaining cell homeostasis and genomic stability, and its inactivation almost always leads to cancer (2-4). Recently, PTEN has been proposed to conform to the continuum model of tumor suppression (5). Rather than following discrete and stepwise changes in PTEN allele loss as described in the two-hit hypothesis, increasing evidence supports dosage-dependency of PTEN tumor suppressor function. A tight correlation between PTEN expression level and function has been demonstrated using a series of targeted PTEN alleles which resulted in varying levels of PTEN expression in mice. Data from these studies show that a subtle $20 \%$ reduction in PTEN protein levels may already lead to deregulated signaling and subsequent development of sporadic tumors, albeit to a lesser extent compared to mice with heterozygous knockout of PTEN (6). However, further downregulation may be required for the disease to progress (7).

Quasi-insufficiency of PTEN can be caused by rare mutations, promoter hypermethylation and antisense regulation. Additionally, post-transcriptional regulation by oncogenic microRNAs (miRNAs/miRs) has been shown to subtly decrease PTEN levels by binding to the 3'untranslated region (3'UTR) $(8,9)$. In colorectal cancer, which has a low rate of PTEN coding region mutations, dysregulation by miRNAs appears to be the main cause of PTEN inactivation, accounting for $\sim 70 \%$ of cases (4). While it may seem unlikely that these miRNAs alone are able to downregulate PTEN levels enough to allow cancer progression, studies have shown that these 
miRNAs can simultaneously modulate the levels of transcription factors in the nucleus, directly or indirectly, to augment downregulation of their target proteins $(10,11)$. Furthermore, individual miRNAs can simultaneously downregulate several tumor suppressor mRNA targets, leading to a single phenotypic readout (12-15). As certain miRNAs only require 6-8 bp of perfect complementarity, they can bind to multiple and otherwise unrelated transcripts and form competing endogenous RNA (ceRNA) networks (16). Thus, a physiologically relevant PTEN miRNA should be able to negatively regulate a ceRNA transcript or network that sustains PTEN transcript levels.

Extensive investigation of the PTEN ceRNA network has revealed transcripts that can act as potent PTEN derepressors (17-19). The majority of these ceRNAs have not been functionally linked to PTEN mRNA before, but are able to act as molecular sponges that can sequester and inhibit shared miRNAs from inactivating PTEN (16). These PTEN ceRNAs included PTEN1P, TNRC6B, VCAN and ZEB2, all of which were able to rescue PTEN expression upon overexpression of either their transcripts or their isolated 3'UTR fragments (17-19). The functional characterization of ceRNAs as derepressors of the PTEN transcript has helped clarify the poorly-understood crosstalk mediated by the 3'UTR of the mRNA, and the specific nuances that PTEN expression levels manifest in cancer, either via their synergistic or antagonistic interactions with other PTEN-inactivating mechanisms (18).

The present study aimed to identify other PTEN ceRNAs that may modulate its tumor suppressive function and validate the functional relevance of the putative ceRNAs.

\section{Materials and methods}

Cell line maintenance and transfection. HCT116 colorectal carcinoma cells were sourced from the American Type Culture Collection. HCT116 cells were cultured in Roswell Park Memorial Institute 1640 (RPMI-1640) medium supplemented with $10 \%$ fetal bovine serum (FBS) (Gibco; Thermo Fisher Scientific, Inc.), $50 \mathrm{U} / \mathrm{ml}$ penicillin/streptomycin and $2 \mathrm{~g} / 1$ sodium bicarbonate. All transfection experiments were performed using Lipofectamine ${ }^{\circledR} 2000$ (Invitrogen; Thermo Fisher Scientific, Inc.) according to the manufacturer's instructions. The amount of plasmid and volume of Lipofectamine 2000 was optimized to achieve $70-80 \%$ transfection efficiency for all functional and molecular characterization experiments. A ratio of $1 \mathrm{ng}$ plasmid per 100 cells was used to transfect cells that were seeded $24 \mathrm{~h}$ earlier. Transfection efficiency for the overexpression constructs was assessed through parallel transfection with pmR-ZsGreen1 empty vector (Clontech Laboratories, Inc.) and fluorescence imaging, while knockdown efficiency of siRNA experiments was assessed via RT-qPCR. miRNA overexpression set-ups included pmR-ZsGreen1 empty vector control and pmR-ZsGreen1-miR4465, whereas 3'UTR overexpression set-ups included pmiRGLO empty vector control, and the different 3'UTRs cloned into the pmiRGLO vector, namely: pmiRGLO-DNMT3B, pmiRGLO-TET3, and pmiRGLO-PTEN. Daily transfection of the siRNAs using their optimized concentrations were performed for the knockdown experiments. Cells were maintained in a $37^{\circ} \mathrm{C}$ humidified incubator with $5 \% \mathrm{CO}_{2}$.

Antibodies and plasmid constructs. The rabbit polyclonal anti-PTEN (cat. no. PA5-27304; 1:1,000) and rabbit polyclonal anti-TET methylcytosine dioxygenase 3 (TET3; cat. no. PA5-31860; 1:1,000) antibodies were from Invitrogen (Thermo Fisher Scientific, Inc.). The mouse monoclonal anti-GAPDH (cat. no. CB1001; 1:1,500) antibody was from EMD Millipore. The rabbit monoclonal anti-DNA methyltransferase $3 \beta$ (DNMT3B; cat. no. 67259; 1:1,000) antibody was from Cell Signaling Technology, Inc. The goat anti-rabbit $\operatorname{IgG}(1: 5,000$; cat. no. 31460) and goat anti-mouse IgG (H+L) (1:10,000; cat. no. 31430) secondary antibodies conjugated with horseradish peroxidase were obtained from Invitrogen (Thermo Fisher Scientific, Inc.).

The 3'UTR of human PTEN (NM_001304717), DNMT3B (NM_006892.3), TET3 (NM_001287491.1) and the pre-miR-4465 gene (NR_039675.1) were amplified by PCR from human genomic DNA using primers listed in Table I. The wild-type 3'UTR fragments were cloned into the pmirGLO dual-luciferase miRNA target expression vector (Promega Corporation), downstream of the firefly luciferase reporter. A mutant version of the 3'UTRs was generated by site-directed mutagenesis of the wild-type 3'UTRs in pmirGLO, in which a TG $>$ AC substitution within the miR-4465 seed sequence binding site (5'-ACTACAA-3') was introduced through splicing-by-overlap-extension PCR using the primers listed in Table I.

The miR-4465 precursor primers were designed to amplify a genomic DNA fragment that includes 206 bp upstream and 226 bp downstream of the pri-miR-4465 sequence. The amplicon was cloned into the mammalian miRNA expression vector pmR-ZsGreen1 (Clontech Laboratories, Inc.).

The PCR reaction mixture contained a final concentration of 1X Titanium ${ }^{\circledR}$ Taq PCR buffer (Clontech Laboratories, Inc.), $0.125 \mu \mathrm{M}$ of each deoxynucleoside triphosphate (dNTP) (Promega Corporation), $2 \mu \mathrm{M}$ of each forward and reverse primers, 1X Titanium ${ }^{\circledR}$ Taq polymerase (Clontech Laboratories, Inc.) and human genomic DNA template extracted from HK-2 cells (ATCC ${ }^{\circledR}$ CRL-2190 ${ }^{\mathrm{TM}}$ ) available at the Disease Molecular Biology and Epigenetics Laboratory of the National Institute of Molecular Biology and Biotechnology (Quezon City, Philippines). The PCR program used was as follows: Initial denaturation at $95^{\circ} \mathrm{C}$ for $5 \mathrm{~min}$, followed by 25-30 cycles of denaturation at $95^{\circ} \mathrm{C}$ for $30 \mathrm{sec}$, annealing at $55-65^{\circ} \mathrm{C}$ for $30 \mathrm{sec}$, and extension at $72^{\circ} \mathrm{C}$ for $30-60 \mathrm{sec}$, with a final extension step at $72^{\circ} \mathrm{C}$ for $10 \mathrm{~min}$. All plasmid constructs were verified by sequencing.

$R T$ - $q P C R$. HCT116 cells were seeded at a density of 200,000 cells/well in a 12 -well plate and transfected after $24 \mathrm{~h}$ with the appropriate amount of pmiRGLO constructs, pmRZsGreen 1 constructs, siRNA and target protectors using Lipofectamine 2000. Cells were harvested $48 \mathrm{~h}$ post-transfection and total RNA was extracted using TRIzol ${ }^{\circledR}$ reagent (Invitrogen; Thermo Fisher Scientific, Inc.). For first strand cDNA synthesis, $2 \mu \mathrm{g}$ of total RNA was mixed with $1 \mu \mathrm{l}$ of $50 \mu \mathrm{g} / \mu \mathrm{l}$ random hexamers, $10 \mu \mathrm{M}$ oligo-dT and $15 \mu \mathrm{l}$ diethyl pyrocarbonate (DEPC)-treated sterile distilled deionized 
Table I. Primers used for generation and site-directed mutagenesis of 3'UTR constructs.

\begin{tabular}{|c|c|}
\hline Primer & Primer sequence $\left(5^{\prime} \rightarrow 3^{\prime}\right)$ \\
\hline DNMT3B-3'UTR-F & $\begin{array}{l}\text { ATTAAGGAGCTCGCACAAATCAGAC } \\
\text { CTGGCTGCTTG }\end{array}$ \\
\hline DNMT3B-3'UTR-Fmut & $\begin{array}{l}\text { TTCTCCTAAAACTTTAAAACTACAAG } \\
\text { TAGGTAGCAACGTGGC }\end{array}$ \\
\hline DNMT3B-3'UTR-R & $\begin{array}{l}\text { GTTCTAGAGCTCCCCAGAGGGTTCTA } \\
\text { TTG }\end{array}$ \\
\hline DNMT3B-3'UTR-Rmut & $\begin{array}{l}\text { GCCACGTTGCTACCTACTTGTAGTTTT } \\
\text { AAAGTTTTAGGAG }\end{array}$ \\
\hline TET3-3'UTR-F & $\begin{array}{l}\text { CATGGCGAGCTCTAACAATCAGATGA } \\
\text { CCGCTATAGG }\end{array}$ \\
\hline TET3-3'UTR-Fmut & $\begin{array}{l}\text { GCCTAGATTTAAACAGCAACTACAAA } \\
\text { AAAAAAGTATGTTTTAAC }\end{array}$ \\
\hline TET3-3'UTR-R & $\begin{array}{l}\text { ACTTATTCTAGACCGTGGACAGGGCA } \\
\text { CACTGGGGAG }\end{array}$ \\
\hline TET3-3'UTR-Rmut & $\begin{array}{l}\text { GTTAAAACATACTTTTTTTTTGTAGTT } \\
\text { GCTGTTTAAATCTAGGC }\end{array}$ \\
\hline PTEN-3'UTR-F & $\begin{array}{l}\text { AAGAGGGATAAAACACCATGAAAAT } \\
\text { AAACTACAATAAACTGAAAATG }\end{array}$ \\
\hline PTEN-3'UTR-Fmut & $\begin{array}{l}\text { TTAACTGTTAGGGAATTTTACTACAA } \\
\text { TACTGAATACATATAATG }\end{array}$ \\
\hline PTEN-3'UTR-R & GCGGAGCTCGAGCACATGTAGGACAATTTCTACTG \\
\hline PTEN-3'UTR-Rmut & $\begin{array}{l}\text { CATTATATGTATTCAGTATTGTAGTA } \\
\text { AAATTCCCTAACAGTTAA }\end{array}$ \\
\hline
\end{tabular}

DNMT3B, DNA methyltransferase 3 $\beta$; TET3, TET methylcytosine dioxygenase 3; UTR, untranslated region; F, forward; R, reverse; mut, mutant.

$\mathrm{H}_{2} \mathrm{O}\left(\mathrm{sddH}_{2} \mathrm{O}\right)$. This was incubated in a thermocycler for $5 \mathrm{~min}$ at $70^{\circ} \mathrm{C}$ then immediately placed on ice. The following were then added to make up a total volume of $25 \mu \mathrm{l}: 5 \mu \mathrm{l}$ 5X M-MLV RT buffer, $1.5 \mu 110 \mathrm{mM}$ dNTPs, $0.625 \mu \mathrm{l} 40 \mathrm{U} / \mu \mathrm{l}$ RNAsin ribonuclease inhibitor, $1.0 \mu \mathrm{l}$ M-MLV RT (200 U/ $\mu \mathrm{l}$; Promega Corporation) and $1.875 \mu \mathrm{l}$ DEPC-treated $\mathrm{sddH}_{2} \mathrm{O}$. The first strand cDNA samples were then used as template for RT-qPCR.

PowerUp $^{\mathrm{TM}} \mathrm{SYBR}^{\circledR}$ Select Master mix (Invitrogen; Thermo Fisher Scientific, Inc.) was used for RT-qPCR following the manufacturer's protocol. For statistical analysis of gene expression, triplicate reactions were prepared per template per primer pair. Adjustment of the baseline and threshold values determined the threshold cycles for the amplification curves. Relative quantification was done with the use of generated standard curves per gene target. Target gene expression was normalized against cyclophilin expression, and fold-change expression relative to the untransfected control/empty vector control was calculated (20). Primers used for RT-qPCR are summarized in Table II.

Absolute quantification using Quantigene analysis. Quantigene miRNA Assay (Affymetrix; Thermo Fisher Scientific, Inc.) was used for absolute quantification of miR-4465 in HCT116 cells. Since there is no required RNA
Table II. RT-qPCR primers used for detecting DNMT3B, TET3 and PTEN mRNA.

\begin{tabular}{ll}
\hline Primer & \multicolumn{1}{c}{ Sequence $\left(5^{\prime} \rightarrow 3^{\prime}\right)$} \\
\hline DNMT3B-qRT-F & GAATTACTCACGCCCCAAGGA \\
DNMT3B-qRT-R & ACCGTGAGATGTCCCTCTTGTC \\
PTEN-qRT-F & CGTATTCTGTAGGTAATCTCTG \\
PTEN-qRT-R & GGTGATGCCTTCAAGTAAC \\
TET3-qRT-F & CAGAAGGAGAAGCTGAGCAC \\
TET3-qRT-R & AGCACCGAGTAGCTCTCCAC \\
Cyclophilin A-F & CCTAAAGCATACGGGTCCTGGCATC \\
Cyclophilin A-R & GTGGAGGGGTGCTCTCCTGAGCTAC
\end{tabular}

DNMT3B, DNA methyltransferase 3 $\beta$; TET3, TET methylcytosine dioxygenase 3; RT-qPCR, reverse transcription-quantitative PCR; $\mathrm{F}$, forward; $\mathrm{R}$, reverse.

extraction and cDNA synthesis step, miRNA quantification is more reliable compared with RT-qPCR using TaqMan probes due to minimal loss of RNA yield. This branched DNA signal amplification technology is a hybridization-based assay that relies on using miRNA-specific probes for normalization. 
Functionally validated pre-designed probes with high sensitivity and specificity for miR-4465 were obtained from eBioscience (Affymetrix; Thermo Fisher Scientific, Inc.). The miR-4465 probe (5'-CUCAAGUAGUCUGACCAGGGGA-3') was determined bioinformatically by Affymetrix to be specific to human samples and to have low cross-reactivity towards closely related miRNA family members.

Cultured cells were lysed to release the miRNAs to be stabilized by overnight hybridization in a 96-well plate with capture extenders, label extenders and target-specific capture probes from the Quantigene miRNA assay kit. Pre-amplifier molecules were added to allow hybridization to each pair of gene-specific probe sets for signal amplification. Hybridization of amplifier molecules with pre-amplifiers was performed prior to probing with alkaline phosphatase-conjugated oligonucleotides. Generation of a luminescent signal proportional to the amount of target miRNA present in each sample was achieved upon addition of a chemilumigenic substrate to each well. Luminescent signal was detected using the FLUOstar Omega microplate reader (BMG Labtech GmbH). For statistical analysis, triplicates of cell lysates per set-up were used to calculate assay precision. For absolute quantitation, a standard curve was generated for the samples of known miRNA concentrations, and this was used as reference to calculate the miRNA concentration for each sample.

siRNA design and optimization. Pre-designed siRNA oligonucleotides directed against all transcript variants of human PTEN, DNMT3B and TET3 were obtained from Qiagen (Qiagen Sciences, Inc.) as follows: PTEN, Product ID Hs_TET3_2, GeneGlobe cat. no. SI05784268, target sequence: CTCGCGCGGCATGGTATGAAA; DNMT3B, Product ID Hs DNMT3B_2, GeneGlobe cat. no. SI00092967, target sequence: AAGGACTACTTTGCATGTGAA; TET3, Product ID Hs_PTEN_6, GeneGlobe cat. no. SI00301504, target sequence: AAGGCGTATACAGGAACAATA. The siRNAs were designed to specifically target their corresponding transcripts. Designed with asymmetrical 5'base pair stabilities, these double-stranded oligos ensure entry of less tightly-bound antisense strand to the RNA-induced silencing complex while reducing risk of off-target effects by degrading the sense strand, which can be incorrectly processed by the RNA-induced silencing complex.

To optimize the amount of siRNA to be used for transfections, different amounts of gene-specific siRNA or the AllStars negative control siRNA (cat. no. 1027281; Qiagen Sciences, Inc.) were tested. Based on the recommended optimal concentration for knockdown, which is $5-30 \mathrm{nM}$, the concentrations of 5, 15, 30 and $60 \mathrm{nM}$ were used. RNA extraction with TRIzol (Invitrogen; Thermo Fisher Scientific, Inc.) of siRNA-transfected cells was performed according to the manufacturer's protocol, and the generated corresponding cDNA was used for RT-qPCR to validate the efficacy of the siRNAs in decreasing individual DNMT3B, TET3 and PTEN transcript levels. The optimum concentration for each siRNA was $30 \mathrm{nM}$.

Dual-luciferase reporter assay. HCT116 cells were seeded at a density of 10,000 cells/well in a 96-well plate and transfected after $24 \mathrm{~h}$. For the endogenous dual-luciferase assays, cells were transfected with $200 \mathrm{ng}$ of empty pmirGLO vector (Promega
Corporation), pmirGLO-PTEN-3'UTR-wild type (wt)- or mutant (mut) 4465, pmirGLO-DNMT3B-3'UTR-wt/mut4465 and pmirGLO-TET3-3'UTR-wt/mut4465. For the miRNA co-transfection dual-luciferase assays, cells were co-transfected with 20 ng empty pmirGLO vector, pmirGLO-PTEN3'UTR-wt/mut4465, pmirGLO-DNMT3B-3'UTR-wt/mut4465 or pmirGLO-TET3-3'UTR-wt/mut4465 together with $200 \mathrm{ng}$ empty pmR-ZsGreen1 vector (Clontech Laboratories, Inc.) or pmR-ZsGreen-miR-4465 construct. The construct preparations were complexed with Lipofectamine 2000 for transfection of cells in triplicate wells. Luciferase activity was measured $48 \mathrm{~h}$ post-transfection using the Dual-Luciferase ${ }^{\circledR}$ Reporter Assay System (Promega Corporation) and the FLUOstar Omega microplate reader (BMG Labtech $\mathrm{GmbH}$ ). The supernatant of the centrifuged lysates was sequentially added with LARII (Luc2 substrate) and Stop and GLO reagents (Rluc substrate) with a 12 -sec timeframe of a continuous read. Fast kinetics function of the multi-mode reader was utilized to continuously detect luminescence. Degree of miRNA regulation was calculated by dividing the sum of the RLUs of the Luc2 interval $(0.5$ to $12 \mathrm{sec})$ with that of the Rluc interval $(12.5$ to $24 \mathrm{sec})$. Data are expressed as the mean values of normalized firefly relative luciferase units (RLUs) per set-up; raw firefly RLUs were normalized against the internal control Renilla luciferase RLUs in each well prior to normalizing against the empty vector control.

Target protection experiments. Target protector oligonucleotides specific to the respective miR-4465 binding site and flanking sequences within the 3'UTR of the PTEN, DNMT3B or TET3 3'UTRs were co-transfected with pmR-ZsGreen1-miR-4465 into HCT116 cells. The target protectors were designed using the Qiagen miRNA Target Protector design tool (https://www.qiagen. $\mathrm{com} / \mathrm{ph} /$ shop/genes-and-pathways/custom-products/customassay-products/custom-mirna-products/\#target-protector) with the RefSeq ID of PTEN transcript variant 1 (NM_000268), DNMT3B transcript variant 1 (NM_006892.3) or TET3 transcript variant 1 (NM_001287491.1) as reference templates. Selection of the 40-bp context sequences was performed by including 20 -bp sequences flanking the respective miRNA binding site in the 3'UTRs of the target MREs. An effective concentration of $200 \mathrm{nM}$ miR-4465 target protector co-transfected with ZsG-miR-4465 was determined through preliminary transfection optimization experiments. Target protector efficiency for miRNA inhibition was measured using dual luciferase assays or RT-qPCR from transfected cells vs. control setups.

Western blotting. Seeding and transfection of HCT116 cells in 12-well plates were performed as described for RT-qPCR. Total protein was extracted from cells $48 \mathrm{~h}$ post-transfection using radioimmunoprecipitation lysis buffer $(150 \mathrm{mM} \mathrm{NaCl}$, $0.5 \%$ sodium deoxycholate, $0.1 \%$ SDS and $50 \mathrm{mM}$ Tris $\mathrm{pH} 8.0$ ) supplemented with protease inhibitors $(1 \mathrm{mM}$ phenylmethylsulfonyl fluoride, $5 \mathrm{mM}$ EDTA and $10 \mu \mathrm{M}$ E64; Roche Diagnostics $\mathrm{GmbH}$ ). Lysates were centrifuged at 10,000 x g for $20 \mathrm{~min}$ at $4^{\circ} \mathrm{C}$ and transferred to fresh tubes. Total protein concentration was measured using the bicinchoninic acid assay. Protein extracts were snap-frozen in liquid nitrogen 
and stored at $-80^{\circ} \mathrm{C}$ until use. For polyacrylamide gel electrophoresis, $30 \mu \mathrm{g}$ of total protein per setup was loaded into 4-15\% Mini-PROTEAN ${ }^{\circledR}$ TGX Stain-Free ${ }^{\mathrm{TM}}$ Protein Gels (Bio-Rad Laboratories, Inc.). Semi-dry electroblot transfer of proteins onto polyvinylidene fluoride membranes was done at $2.5 \mathrm{~mA}, 25 \mathrm{~V}, 10 \mathrm{~min}$ using the Trans-Blot ${ }^{\circledR}$ Turbo Blotting system (Bio-Rad Laboratories, Inc.). Blocking of membranes was performed using $5 \% \mathrm{w} / \mathrm{v}$ whey protein in Tris-buffered saline with $0.1 \% \mathrm{v} / \mathrm{v}$ Tween-20 (TBST) at room temperature for $1 \mathrm{~h}$. Blots were probed overnight at $4^{\circ} \mathrm{C}$ with the primary antibodies described above and washed with TBST. This was followed by incubation with the appropriate secondary antibody conjugated with horseradish peroxidase (HRP) for $1 \mathrm{~h}$ at room temperature. Bands were visualized with enhanced chemiluminescence (ECL) substrate and imaged using the ChemiDoc Touch Imaging System (Bio-Rad Laboratories, Inc.). Densitometric analysis of digitized band intensities was performed using GelQuant.NET (v1.8.2). Quantified PTEN, DNMT3B, or TET3 gene expression was normalized against GAPDH.

Caspase 3/7 assay. HCT116 cells were seeded at a density of 100,000 cells/well in a 24 -well plate and were transfected at $24 \mathrm{~h}$ post-seeding. After $48 \mathrm{~h}$, the cells were reseeded into a 96-well plate. For the assay proper, trypsinized transfected cells were seeded in two sets of triplicates corresponding to the uninduced and induced groups per transfection set-up. Cells were treated with $5 \mathrm{mM}$ sodium butyrate and incubated further for 16-20 $\mathrm{h}$ for the induction of apoptosis. Following the addition of $10 \mathrm{ml}$ Caspase 3/7 reagent (Promega Corporation) per well, the plate was mixed by shaking at $300 \mathrm{rpm}$ for $30 \mathrm{sec}$ and incubated for $30 \mathrm{~min}$ at room temperature. Luminescence was measured using a multi-mode plate reader (FLUOstar Omega; BMG Labtech $\mathrm{GmbH}$ ), and statistical analysis was performed by comparing the relative luminescent units of the induced and uninduced groups in each set-up.

Wound healing assay. HCT116 cells were seeded at 100,000 cells/well in a 96-well plate. At 24 h, $250 \mathrm{ng}$ pmR-ZsGreen1 empty vector was co-transfected with either the pmiRGLO construct or the desired siRNA using Lipofectamine 2000 to assess the transfection efficiency through GFP fluorescence. Upon reaching $>95 \%$ confluence at $24 \mathrm{~h}$ post-transfection, a thin artificial wound was introduced to the cell monolayer using a sterile pipette tip. Cells were washed once with $1 \mathrm{X}$ phosphate buffered saline (PBS) and maintained in the appropriate media supplemented with 10 or $4 \%$ fetal bovine serum (FBS). Wound closure was monitored at 0 and $16 \mathrm{~h}$ post-scratch by capturing three fields of view per set-up at $x 4$ magnification using the IN Cell Analyzer 6000 High-Content Imaging system (GE Healthcare Life Sciences). Percent open wound area was analyzed using the TScratch software (http://www.cse-lab.ethz.ch/software/) and reported as the mean $\%$ open wound area per set-up relative to $\%$ open wound area upon initial scratching $(\mathrm{t}=0 \mathrm{~h})$. The migration rate was calculated as follows: [(Percentage open wound area $(\mathrm{t}=0)$-(Percentage open wound area $(\mathrm{t}=16)] / 16 \mathrm{~h}$.

Low serum $(4 \%)$ to full serum $(10 \%)$ conditions were used in would healing assays following miRNA sequestration experiments to allow the demonstration of the tumor suppressive effects of PTEN as a result of its derepression.
Total serum deprivation was not performed, as no effects of oncogenic signaling needed to be decoupled from mitogenic stimulation. Cell migration was monitored only up to $16 \mathrm{~h}$ before the known doubling time of HCT116 cells of $\sim 21 \mathrm{~h}$.

In silico analysis. CEFINDER (https://www.oncomir. umn.edu/cefinder/), a tool that identifies mRNAs sharing miRNA binding sites, was used to rank putative PTEN ceRNAs based on shared PTEN miRNA binding sites in their 3'UTR. TargetScanHuman v.7.1 (http://www.targetscan. org/vert_71/) was used to identify miRNAs that can regulate PTEN and its ceRNAs. RNAfold (http://rna.tbi.univie. ac.at/cgi-bin/RNAWebSuite/RNAfold.cgi) was used to assess structural changes in the 3'UTR upon introduction of a mutation to abrogate a miR-4465 binding site. RNAhybrid (https://bibiserv.cebitec.uni-bielefeld.de/rnahybrid) was used to assess miRNA affinity with 3'UTR sequence by calculating the free energy of binding.

Statistical analysis. For statistical analysis, unpaired two-tailed Student's t-test to measure differences between two set-ups was performed. One-way ANOVA with post hoc Tukey's test was used to test significant differences between multiple set-ups. Data from all quantitative experiments are presented as the mean \pm standard deviation (SD). $\mathrm{P}<0.05$ was considered to indicate a statistically significant difference.

\section{Results}

In silico identification of novel PTEN ceRNAs and miR-4465 as a shared microRNA. Initial bioinformatics analysis using CEFINDER identified TET3, which is a demethylase, and DNMT3B, a de novo methyltransferase, as two of the top scoring hits. TET3 ranked second to the validated PTEN ceRNA TNRC6B, which exhibited the highest number of shared miRNA binding sites with PTEN. PTEN shares 21 miRNA binding sites with TET3 and 5 with DNMT3B (Fig. 1A). By contrast, TET1, TET2 and DNMT3A only share 7, 2 and 2 miRNA binding sites with PTEN, respectively. DNMT3L was not included in the analysis as it had no known regulatory miRNAs based on the analysis on TargetScanHuman v.7.1. These findings were further qualified by mapping the shared miRNAs among DNMT3B, TET3 and PTEN that were broadly conserved across all vertebrates. The results demonstrated that PTEN may theoretically share 32 miRNA binding sites with TET3 and 11 with DNMT3B. DNMT3B and TET3 may share 11 miRNA binding sites, whereas all three transcripts may share 6 miRNA binding sites (Fig. 1B).

To validate the result that DNMT3B, TET3 and PTEN form a ceRNA network, a miRNA able to negatively regulate all three genes via their 3'UTRs needed to be identified. CEFINDER identified only two miRNA families that could bind to all three genes, miR-29-abc-3p and miR-26ab-5p/1297/4465. When these were cross-referenced against the 6 shared miRNAs proposed by TargetScanHuman v.7.1, they were also identified as the top two miRNA families predicted to negatively regulate the three genes. Upon further examination of the relative context scores (a metric for predicted efficacy of repression) of the miRNAs among the three genes, the miR-26ab-5p/1297/4465 family had the highest context score 
A
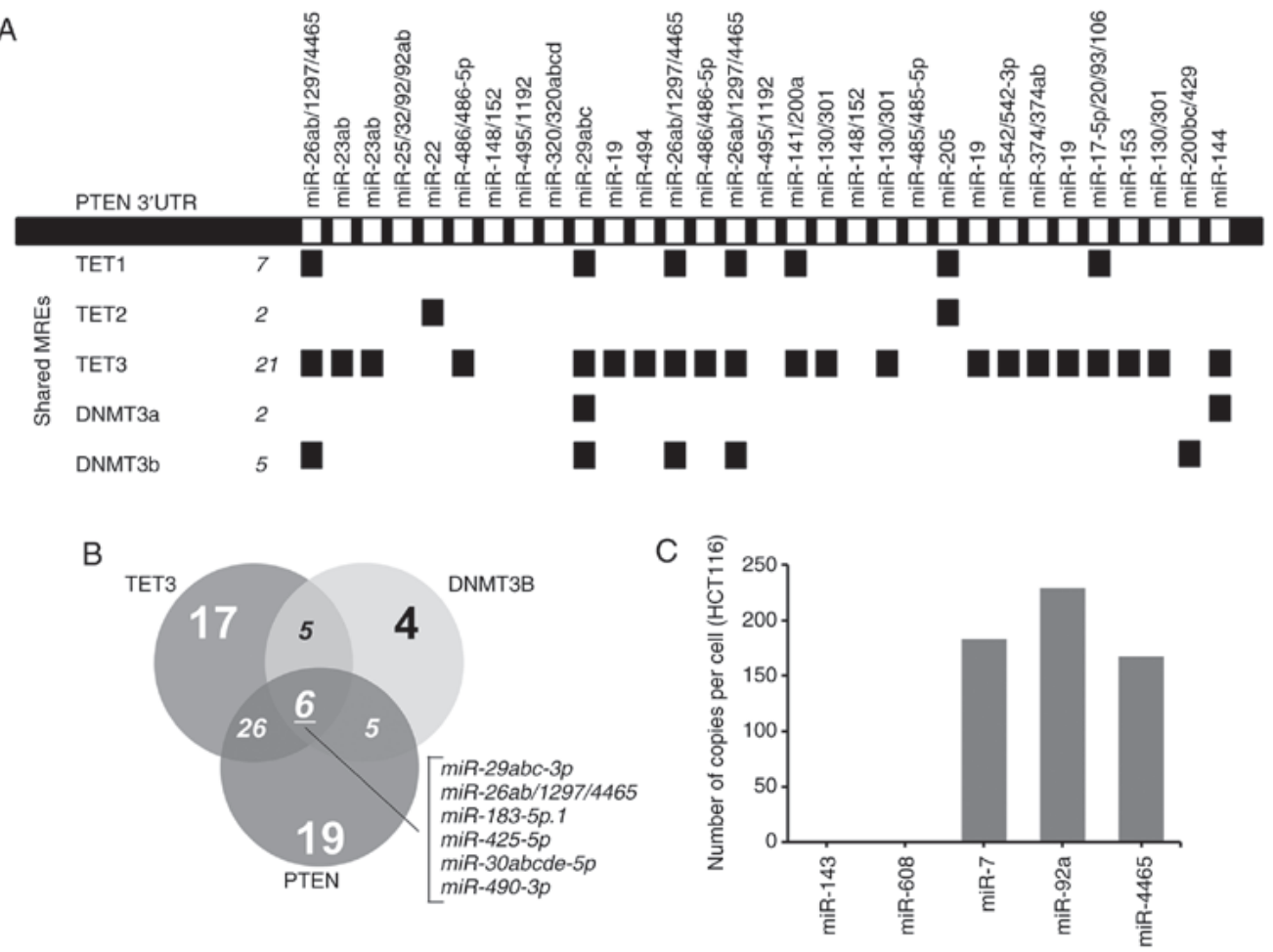

Figure 1. PTEN, TET3 and DNMT3B 3'UTRs share multiple miRNAs. (A) Linear map of PTEN 3'UTR demonstrating the MREs; miRNAs shared with TET1, TET2, TET3, DNMT3a and DNMT3b are indicated below. The map was derived from ceRDB using CEFINDER. (B) Venn diagram of the theoretical number of miRNAs shared among PTEN, TET3 and DNMT3b 3'UTRs determined by TargetScanHuman v.7.1. (C) Number of copies of miR-4465 per cell in HCT116 cells compared with miR-143, miR-408, miR-7 and miR-92a. The expression levels of the mature miRNAs were measured using the Quantigene miRNA assay. miRNA, miR, microRNA; MREs, miRNA response elements; UTR, untranslated region; TET, TET methylcytosine dioxygenase; DNMT3, DNA methyltransferase 3.

for PTEN, but miR-29abc-3p had the highest context scores for DNMT3B and TET3. In addition, the miR26ab-5p/1297/4465 family had a high context score for both PTEN and TET3 and a relatively low context score for DNMT3B. miR-4465 was chosen since it is the only miRNA in this family the role of which in cancer has not been functionally characterized. PTEN and TET3 have four miR-4465 binding sites (according to TargetScanHuman v.7.1; only three according to CEFINDER), whereas DNMT3B only has one. The results of the bioinformatics analysis of miR-4465 binding to TET3, PTEN and DNMT3B 3'UTRs are summarized in Table III.

Previous studies have confirmed the expression of PTEN (21), DNMT3B (22) and TET3 (23) in HCT116 cells. The expression level of miR-4465 in HCT116 cells, however, has not been reported. The results of the Quantigene assays in the present study demonstrated that miR-4465 was present in HCT116 cells at 150-250 copies/cell, which is comparable to the oncogenic miRNAs miR-7 and miR-92a (Fig. 1C).

miR-4465 negatively regulates DNMT3B, TET3 and PTEN via their 3'UTRs. To confirm whether miR-4465 may regulate DNMT3B, TET3 and PTEN individually, precursor miR-4465 was cloned into the miRNA expression vector pmR-ZsGreen1, and fragments of the 3'UTRs of the target genes into the miRNA target expression vector pmirGLO, downstream of a luciferase reporter. Cloned DNMT3B 3'UTR contained one miR-4465 binding site (265-271 bp), cloned TET3 3'UTR contained one (1,958-1,964 bp), whereas cloned PTEN 3'UTR contained two binding sites (41-47 and 1,261-1,268 bp). Quantigene analysis confirmed the overexpression of mature miR-4465 in HCT116 cells transfected with the pmR-ZsGreen1-miR-4465 construct (Fig. S1). Individual overexpression of DNMT3B, TET3 and PTEN 3'UTRs led to a decrease in the FLuc/Rluc ratio in luciferase assays compared with the pmirGLO empty vector set-up (Fig. 2A). These results suggested the negative endogenous regulation of the three genes in HCT116 cells. To implicate miR-4465 in the observed downregulation, pmRZsGreen1-miR-4465 was co-transfected with the individual pmirGLO constructs containing DNMT3B, TET3 and PTEN 3'UTR fragments. Dual luciferase reporter assay revealed that miR-4465 negatively regulated DNMT3B, TET3 and PTEN 3'UTRs in a dose-dependent manner (Fig. 2B).

To further demonstrate that miR-4465 may directly bind to the 3'UTRs of DNMT3B, TET3 and PTEN, each miR-4465 binding site seed sequence (5'-ACTTGAA-3') in the cloned 3'UTR fragments was disrupted via site-directed mutagenesis with a TG $>$ AC substitution. In silico analysis revealed that these mutations changed the secondary structure of each of the 3'UTRs only minimally, while making miR-4465 free energy of binding less negative (Fig. 2C), which was indicative of weaker pairing between the miRNA and the target. Dual luciferase assays using cells transfected with the mut 3'UTRs exhibited an increase in Fluc/Rluc ratio compared with the wt 3'UTR controls (Fig. 2D). These results suggested that the destruction of the binding site partially derepressed the 3 'UTRs from endogenous negative regulation due to the loss of binding by miR-4465. In addition, no dose-dependent negative regulation by miR- 4465 was observed when the mutant 3'UTRs were used for the dual luciferase assays 
Table III. Features of mir-4465 binding onto DNMT3B, TET3 and PTEN 3'UTRs in terms of number of binding sites, total context score and validation from previous TargetScan publications.

\begin{tabular}{|c|c|c|c|c|c|}
\hline Authors, year & Gene & $\begin{array}{l}\text { No. of MREs } \\
\text { within 3'UTR }\end{array}$ & MRE positions & $\begin{array}{c}\text { Cumulative } \\
\text { weighted context } \\
\text { score }^{\mathrm{a}}\end{array}$ & $\begin{array}{c}\text { TargetScan } \\
\text { publication(s) }\end{array}$ \\
\hline Garcia et al., 2011 & PTEN & 4 & $\begin{array}{l}41-47,1261-1268, \\
2619-2626,3800-3807\end{array}$ & -0.76 & (47) \\
\hline $\begin{array}{l}\text { Garcia et al., 2011; } \\
\text { Friedman et al., } 2009\end{array}$ & TET3 & 4 & $\begin{array}{l}1958-1964,4662-4668, \\
5570-5577,5607-5714\end{array}$ & -0.48 & $(47,48)$ \\
\hline Garcia et al., 2011 & DNMT3B & 1 & $265-271$ & -0.09 & (47) \\
\hline
\end{tabular}

(Fig. 2E). These results confirmed that miR-4465 contributed to the negative regulation of DNMT3B, TET3 and PTEN by directly binding to their 3'UTRs, since mutating the binding site abrogated the observed dose-dependent negative regulation exerted by miR-4465 on the three genes.

DNMT3B, TET3 and PTEN are endogenous targets of $m i R-4465$. To determine whether miR-4465 acted on its putative endogenous targets, the effects of its overexpression on DNMT3B, TET3 and PTEN transcript and protein levels in HCT116 were examined using RT-qPCR and western blot analyses, respectively. TET3 and PTEN transcript levels decreased, whereas DNMT3B transcript levels were unchanged in cells overexpressing miR-4465 compared with cells transfected with an empty vector (Fig. 3A). A decrease in the protein levels of all three genes was also observed following miR-4465 overexpression (Fig. 3B and C). These results suggested that miR-4465 may negatively regulate the expression of endogenous TET3 and PTEN via transcript degradation, and DNMT3B via translational repression.

To confirm that the observed endogenous downregulation was due to direct binding of miR-4465 to its target mRNAs, target protectors (TPs) specific to the miR-4465 binding site of each 3'UTR were used in subsequent transfections. Unlike anti-miRs, TPs are locked nucleic acids that can block the interaction of a miRNA with a single target, but leaves other targets of the same miRNA in other transcripts unaffected. Dual luciferase assay results demonstrated derepression of the three 3'UTRs from endogenous miRNA binding upon transfection of their respective TPs in a dose-dependent manner (Fig. 3D). In addition, co-transfection of specific TPs with individual 3'UTR constructs and miR-4465 inhibited the repressive effect of miR-4465 (Fig. 3E-G). The target protectors were only able to inhibit miR-4465 binding to their specific 3'UTR targets.

Evaluation of the DNMT3B, TET3 and PTEN transcript levels upon co-transfection of miR-4465 and the respective TPs was performed to determine whether miR-4465 may downregulate endogenous DNMT3B, TET3 and PTEN levels by direct binding to their 3'UTRs. TET3 and PTEN transcripts were rescued from downregulation by miR-4465; DNMT3B transcript levels, on the other hand, were not affected by
miR-4465 overexpression, consistent with results presented in Fig. 3A and B, suggesting translational repression. The DNMT3B transcript levels remained unchanged following transfection with DNMT3B 3'UTR-specific TPs (Fig. 3H).

DNMT3B, TET3 and PTEN positively modulate each other's expression by competitive miRNA sequestration. To demonstrate possible miRNA-mediated crosstalk among PTEN, DNMT3B and TET3, the effects of shared miRNA sequestration were examined via gene knockdown and 3'UTR overexpression experiments. For knockdown experiments, $30 \mathrm{nM}$ gene-specific siRNA was used, which resulted in knockdown efficiencies of $\geq 60 \%$ (Fig. S2). Knockdown of DNMT3B led to a decrease in the transcript levels of TET3 and PTEN, as identified by RT-qPCR (Fig. 4A). Knockdown of TET3 also led to a decrease in the transcript levels of DNMT3B and PTEN (Fig. 4B). In addition, knockdown of PTEN led to a decrease in the transcript levels of DNMT3B and TET3 (Fig. 4C). These results demonstrated that in the context of HCT116 cells, knockdown of their individual transcripts resulted in downregulated expression of their ceRNAs.

To further determine if the observed downregulation was due, at least in part, to competitive miRNA sequestration, the 3'UTR construct from each gene was overexpressed in HCT116 cells. Overexpression of the DNMT3B 3'UTR led to an increase in the transcript levels of TET3 and PTEN in a dose-dependent manner as assessed by RT-qPCR (Fig. 4D). Overexpression of the TET3 3'UTR also led to an increase in the transcript levels of DNMT3B and PTEN compared with the control cells (Fig. 4E). Additionally, overexpression of the PTEN 3'UTR led to an increase in the transcript levels of DNMT3B and TET3 (Fig. 4F). These results demonstrated that the observed downregulation of the endogenous competitors upon knockdown of one gene and the converse upregulation of the endogenous competitors when the 3'UTR of a gene is overexpressed were at least partly miRNA-dependent and coding region-independent.

DNMT3B and TET3 3'UTR overexpression promotes apoptosis and inhibits migration. To test whether competitive miRNA sequestration and the consequent modulation of PTEN levels may elicit phenotypic readouts consistent with 
A
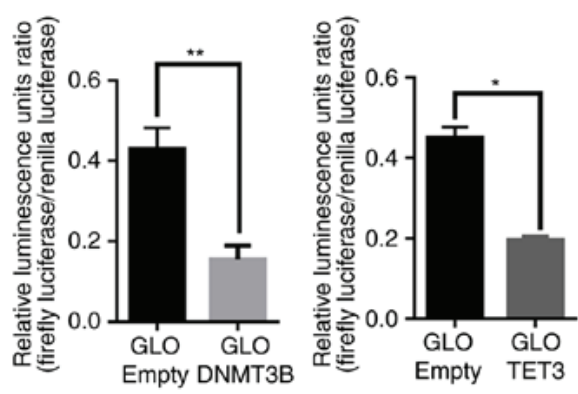

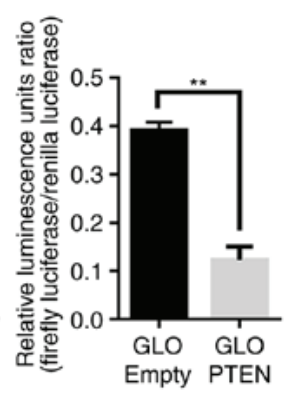

C

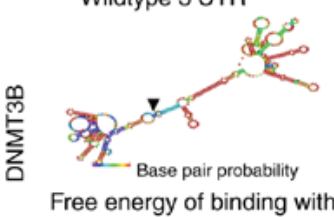

Free energy of binding with miR-4465: "-11.3 $\mathrm{kcal} / \mathrm{mol}$

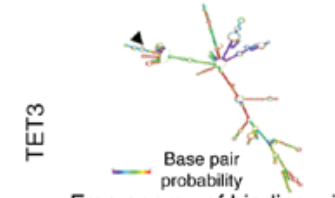

Free energy of binding with miR-4465: *-12.5 kcal/mol

늠

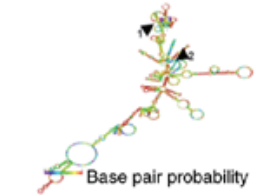

Free energy of binding with miR-4465: $1.7-7 \mathrm{kcal} / \mathrm{mol}$ 2.*-14.4 kcal/mol

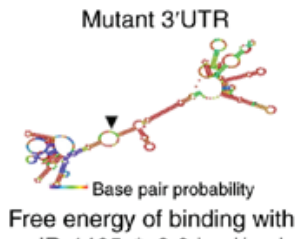

Free energy of binding with miR-4465: *-9.0 kcal/mol

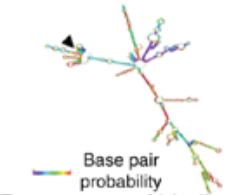

Free energy of binding with miR-4465: *-8.1 kcal/mol

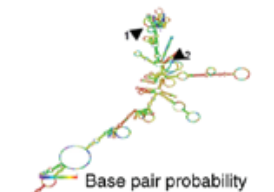

Free energy of binding with $\mathrm{miR}-4465:$ 1. $-6.6 \mathrm{kcal} / \mathrm{mol}$ $2 .-10.1 \mathrm{kcal} / \mathrm{mol}$
B
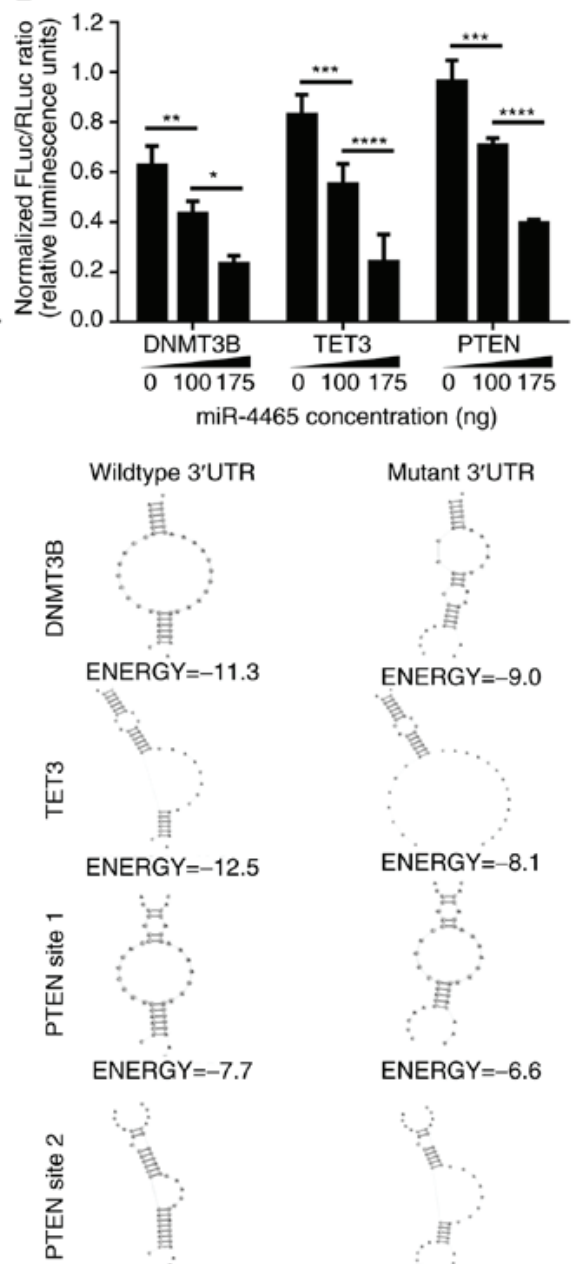

D

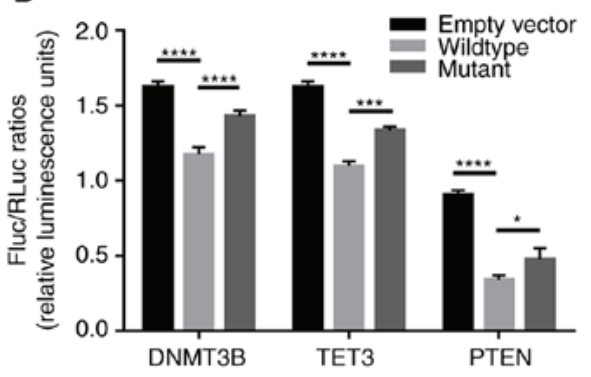

E

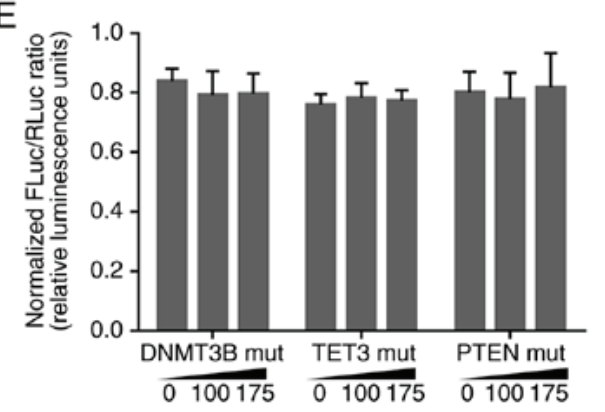

Figure 2. PTEN, TET3 and DNMT3B 3'UTRs are negatively regulated by the shared miRNA-4465. (A) Dual-luciferase assays of HCT1116 cells transfected with an empty vector or pmirGLO-wt DNMT3B-, wt TET3- and wt PTEN-3'UTR demonstrated endogenous downregulation of the transcripts. (B) Dual-luciferase assay results of the dose-dependent downregulation exerted by miR-4465 on DNMT3B, TET3 and PTEN 3'UTRs in HCT116 cells. (C) Secondary structure analyses of DNMT3B, TET3 and PTEN 3'UTRs, and changes in the free energy of binding of miR-4465 to its targets following site-directed mutagenesis of the seed sequence (5'-ACTTGAA-3') with a TG>AC substitution. (D) Dual-luciferase assay results of HCT116 cells transfected with wt or miR-4465 MRE mutant DNMT3B-, TET3- and PTEN-3'UTRs demonstrated endogenous derepression of the mutant transcripts. (E) Dual-luciferase assay results on the abrogation of dose-dependent downregulation exerted by miR-4465 in HTC116 cells co-transfected with miR-4465 MRE mutant 3'UTR constructs. All experiments were performed in cells maintained in $10 \%$ serum. Data are representative of three independent trials and expressed as the mean \pm SD. ${ }^{*} \mathrm{P}<0.05$,

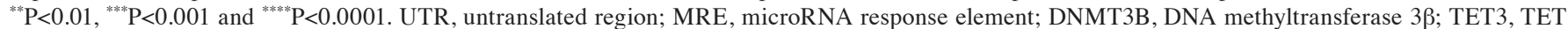
methylcytosine dioxygenase 3; empty, cells transfected with an empty pmirGLO vector; miR, microRNA; MRE, microRNA response element; wt, wild-type.

PTEN derepression, overexpression experiments involving DNMT3B and TET3 3'UTR constructs were performed in full-serum and low-serum conditions. Caspase-Glo 3/7 and wound healing assays were used to determine apoptosis and cell migration, respectively. The results demonstrated the suppression of oncogenesis by DNMT3B and TET3 3'UTRs in a coding-independent and potentially miRNA-dependent manner, specifically by promoting apoptosis and decreasing the migratory capacity in both full-serum (Fig. 5A-C) and low-serum conditions (Fig. 5D-F) compared with the pmiRGLO empty vector control. In all migration assays, the debris observed in the open wound areas were confirmed to be dead cells based on fluorescence microscopy (data not shown). 

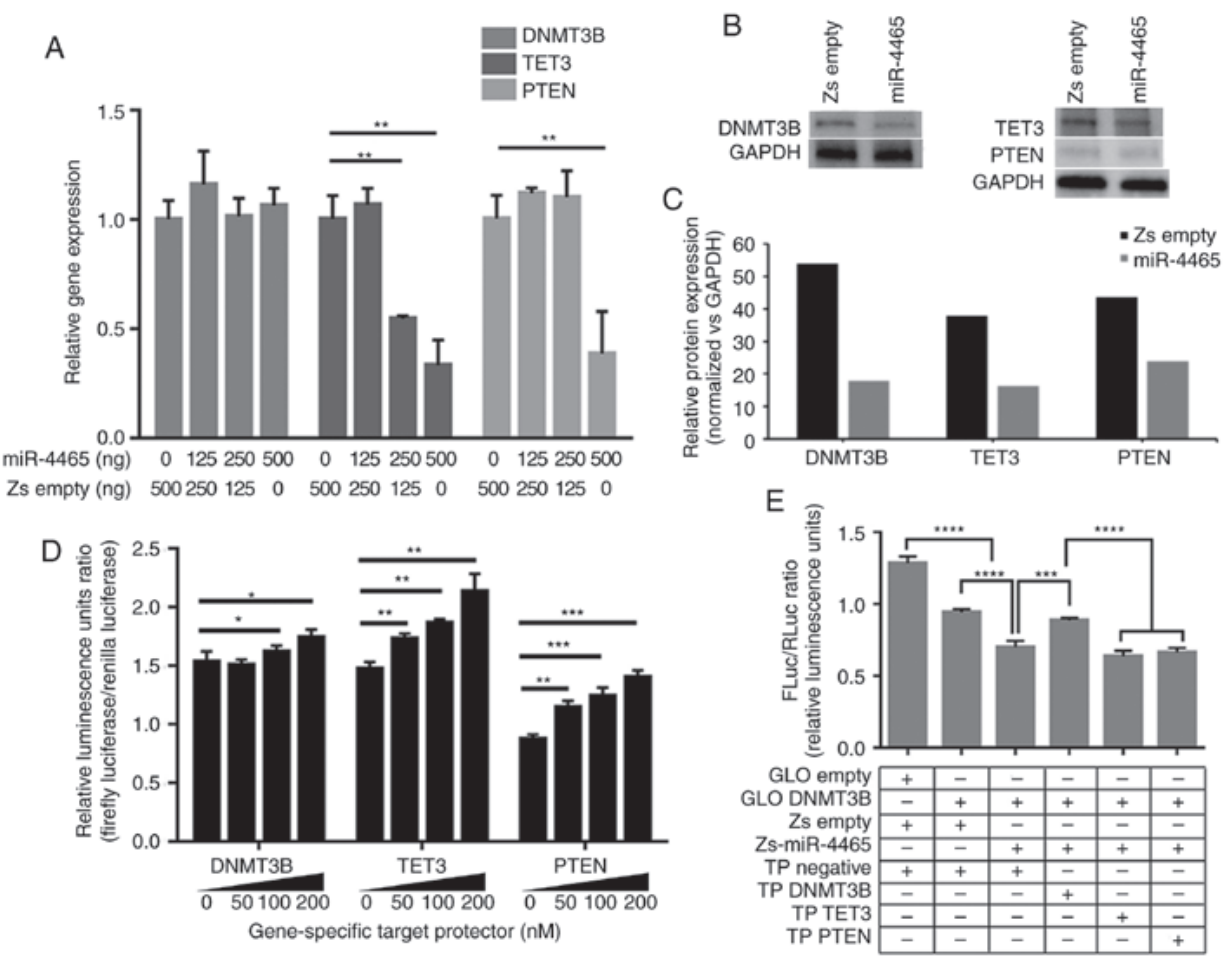

$\mathrm{F}$
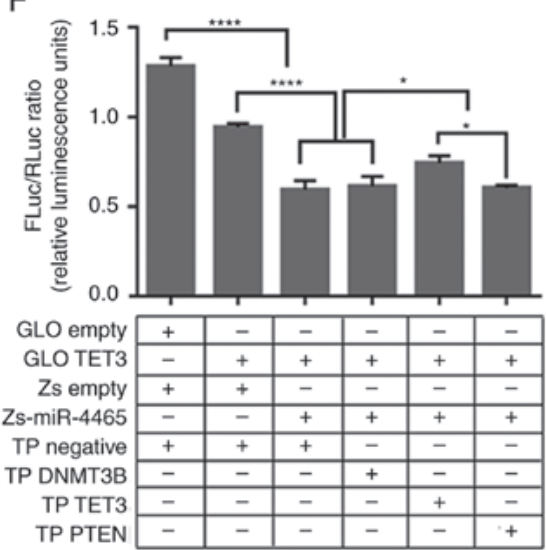

G
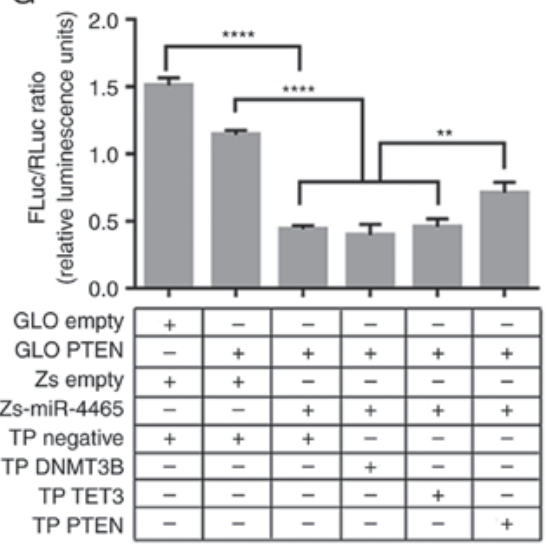

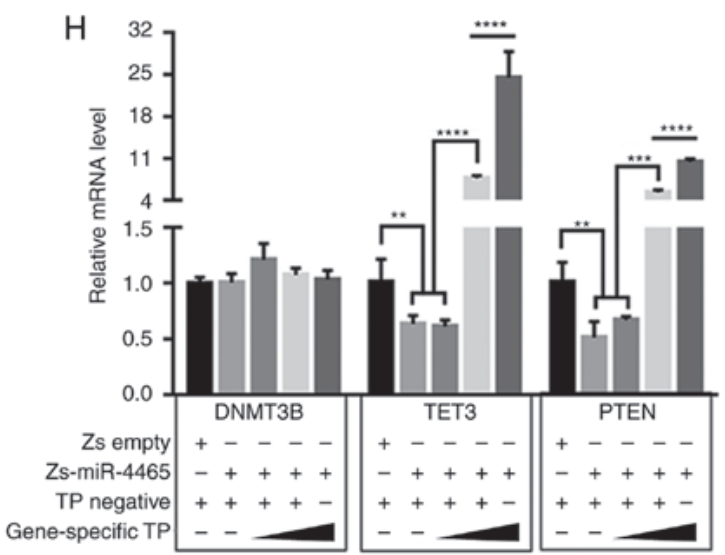

Figure 3. miR-4465 negatively regulates the expression of endogenous DNMT3B, TET3 and PTEN. (A) RT-qPCR demonstrated the effects of increasing amounts of transfected miR-4465 on the endogenous transcript levels of DNMT3B, TET3 and PTEN. (B) Western blot analyses demonstrated the effects of miR-4465 overexpression on endogenous protein levels of DNMT4B, TET3 and PTEN. (C) Relative quantification of the protein expression levels of DNMT3B, TET3 and PTEN in western blots normalized to GAPDH (n=5). (D) Dual-luciferase assays demonstrated derepression of endogenous DNMT3B, TET3 and PTEN following co-transfection with increasing amounts of gene-specific TPs. (E-G) Dual-luciferase assays demonstrated direct targeting of (E) DNMT3B-, (F) TET3- and (G) PTEN-3'UTR by miR-4465 and rescue by gene-specific target protectors. (H) RT-qPCR demonstrated the effects of transfected miR-4465 and co-transfected gene-specific target TPs on the endogenous transcript levels of DNMT3B, TET3 and PTEN. All experiments were performed in cells maintained in $10 \%$ serum. Data presented are representative of three independent trials and expressed as the mean $\pm \mathrm{SD}$. ${ }^{*} \mathrm{P}<0.05,{ }^{* *} \mathrm{P}<0.01$, ${ }^{* * * *} \mathrm{P}<0.001$ and ${ }^{* * * * *} \mathrm{P}<0.0001$. miR, microRNA; UTR, untranslated region; GLO Empty, empty pmirGLO vector; Zs Empty, empty pmR-ZsGreen1 vector; TP, target protector; RT-qPCR, reverse transcription-quantitative PCR; DNMT3B, DNA methyltransferase 3ß; TET3, TET methylcytosine dioxygenase 3. 

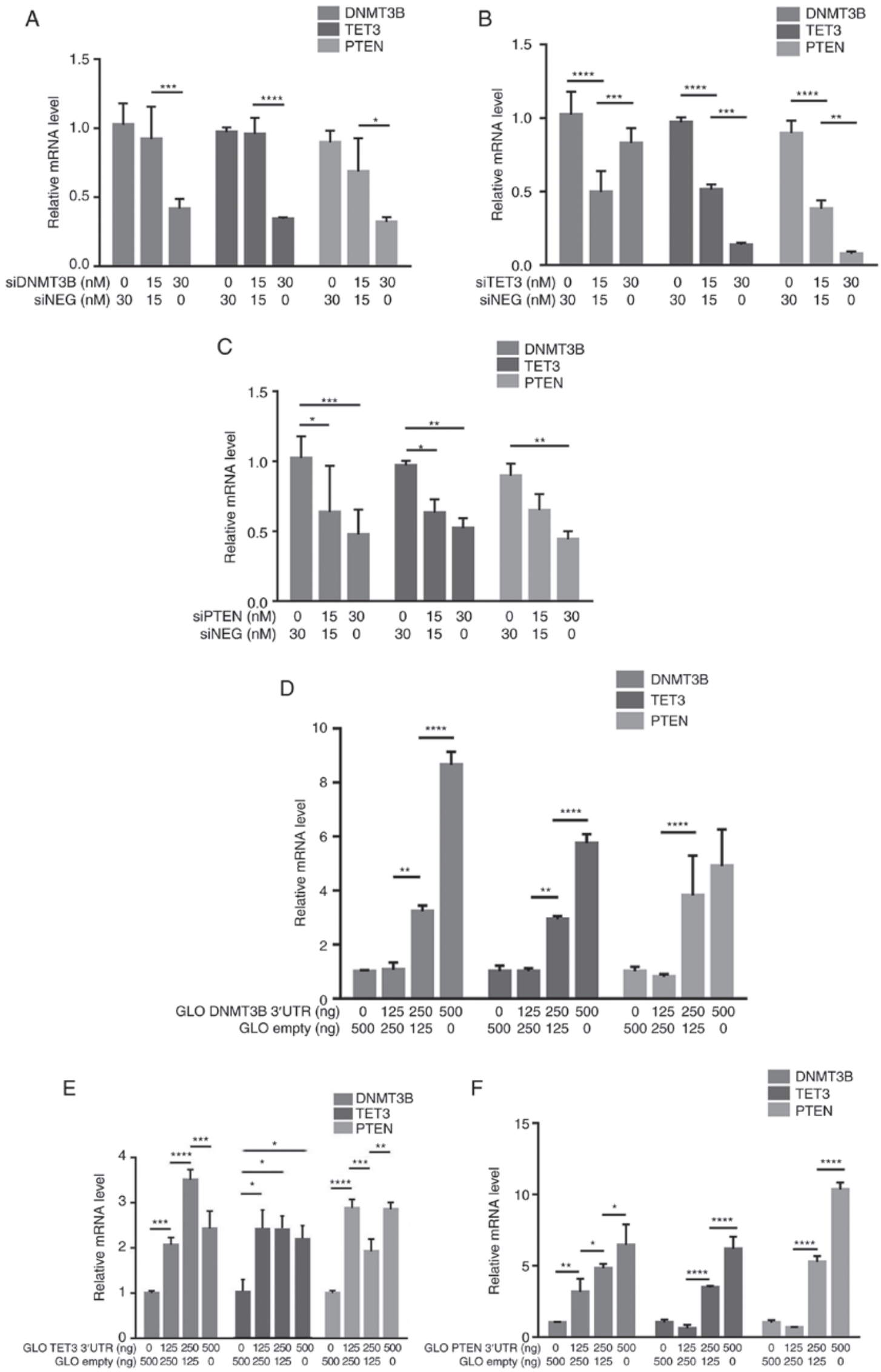

Figure 4. Crosstalk among DNMT3B, TET3 and PTEN through shared miRNA sequestration. (A-C) RT-qPCR demonstrated the reciprocal effects of (A) DNMT3B, (B) TET3 and (C) PTEN knockdown on the transcript levels of their competing endogenous RNAs. (D-F) The effects of overexpression of (D) DNMT3B- (E) TET3- and (F) PTEN-3'UTR on the transcript levels of their competing endogenous RNAs were also assessed by RT-qPCR. All experiments were performed in cells maintained in $10 \%$ serum. Data are representative of three independent trials and expressed as the mean $\pm \mathrm{SD}$. ${ }^{*} \mathrm{P}<0.05,{ }^{* *} \mathrm{P}<0.01$, ${ }^{* * * *} \mathrm{P}<0.001$ and ${ }^{* * * *} \mathrm{P}<0.0001$. UTR, untranslated region; GLO, pmirGLO construct; si-, small interfering RNA; siNEG, negative control small interfering RNA; miRNA, microRNA; DNMT3B, DNA methyltransferase 3 $\beta$; TET3, TET methylcytosine dioxygenase 3; RT-qPCR, reverse transcription-quantitative PCR. 

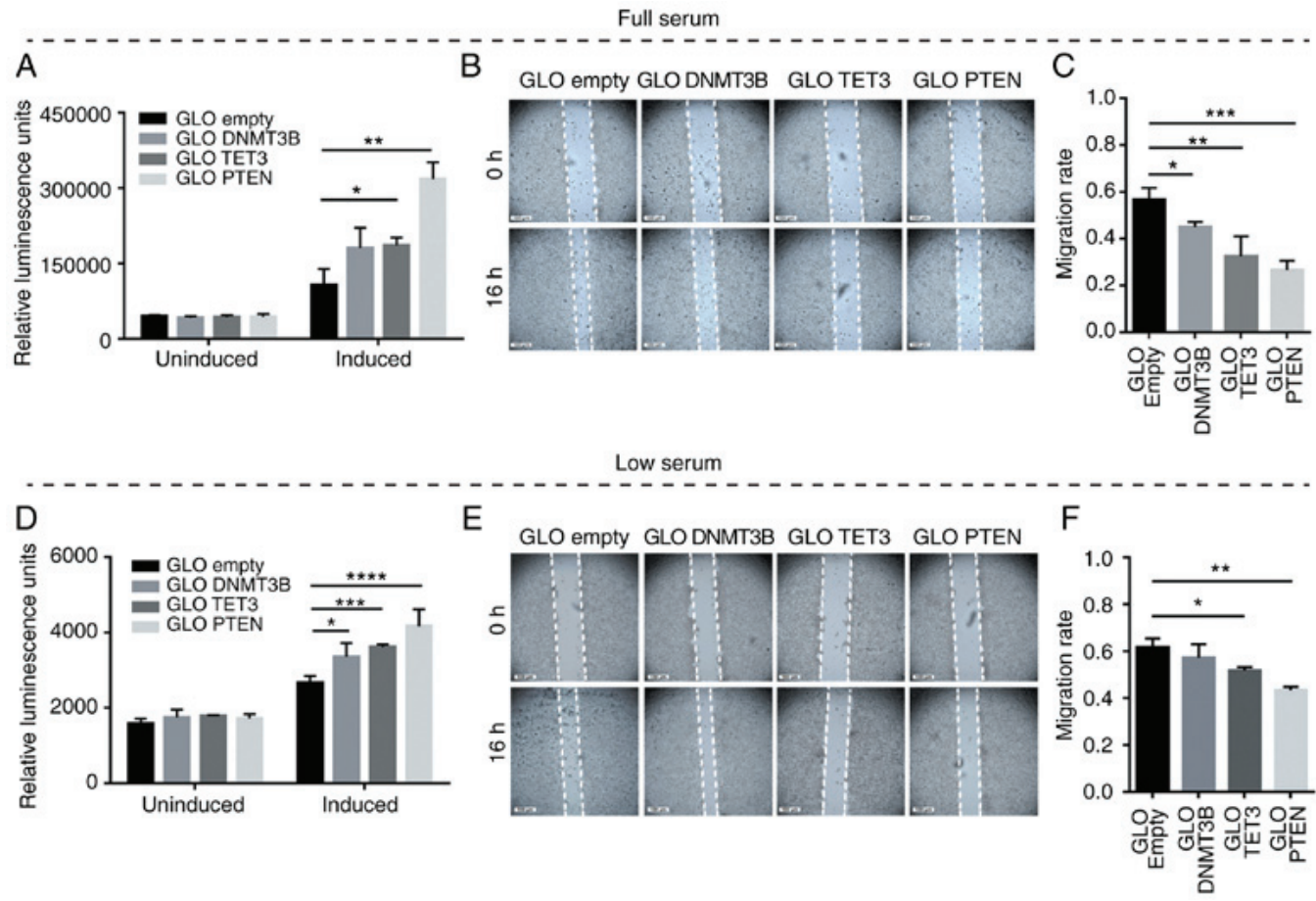

Figure 5. Effects of DNMT3B- and TET3-3'UTR overexpression on apoptosis and cell migration in HCT116 cells. (A and D) Caspase-Glo 3/7 and (B and E) wound healing assays were used to monitor the effects of 3'UTR overexpression on apoptosis and migratory capacity, respectively. (C and F) The migration rates corresponding to (B) and (E). Set-ups A-C and D-F were performed in full-serum (10\%) and low-serum (4\%) conditions, respectively. Data are representative of three independent trials and expressed as the mean $\pm \mathrm{SD} .{ }^{*} \mathrm{P}<0.05,{ }^{* *} \mathrm{P}<0.01,{ }^{* * * *} \mathrm{P}<0.001,{ }^{* * * * *} \mathrm{P}<0.0001$. GLO, pmirGLO construct; empty, GLO empty, pmirGLO empty vector control; DNMT3B, DNA methyltransferase 3 $\beta$; TET3, TET methylcytosine dioxygenase 3.
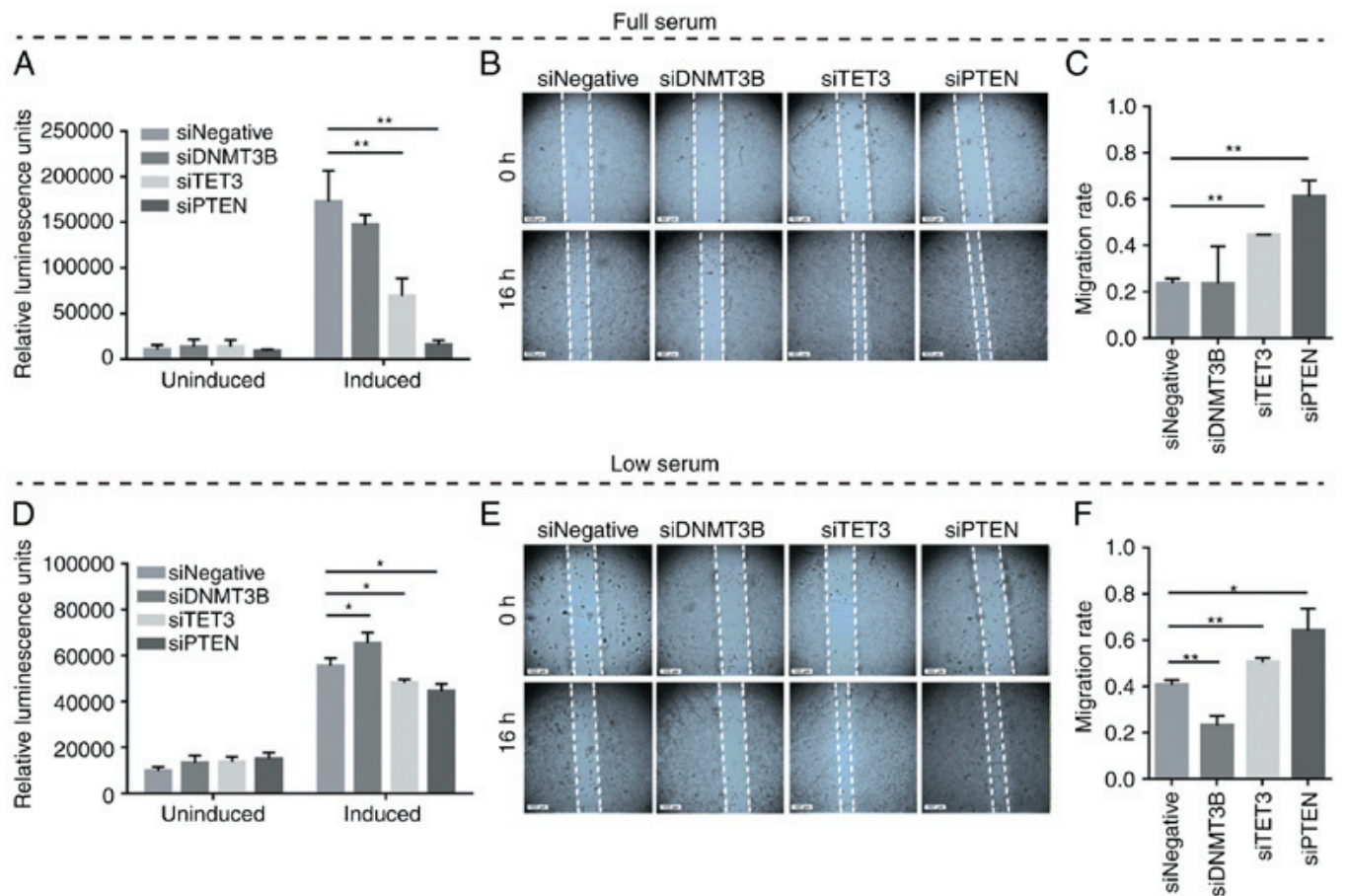

Figure 6. Effects of DNMT3B and TET3 knockdown on apoptosis and cell migration in HCT116 cells. (A and D) Caspase-Glo 3/7 assay and (B and E) wound healing assays were used to monitor the effects of gene knockdown on apoptosis and migratory capacity, respectively. (C and F) The migration rates corresponding to (B and E). Set-ups A-C and D-F were performed in full-serum (10\%) and low-serum (4\%) conditions, respectively. Data presented are representative of three independent trials and expressed as the mean $\pm \mathrm{SD}$. ${ }^{*} \mathrm{P}<0.05,{ }^{* *} \mathrm{P}<0.01$. si-, small interfering RNA; siNEG, negative control small interfering RNA; DNMT3B, DNA methyltransferase 3 $\beta$; TET3, TET methylcytosine dioxygenase 3.

Decoupling the coding-dependent from the miRNA-dependent, coding-independent functions of TET3 and DNMT3B.
TET3 knockdown enhanced migration as well as resistance to apoptosis in both full-serum (Fig. 6A-C) and low-serum 


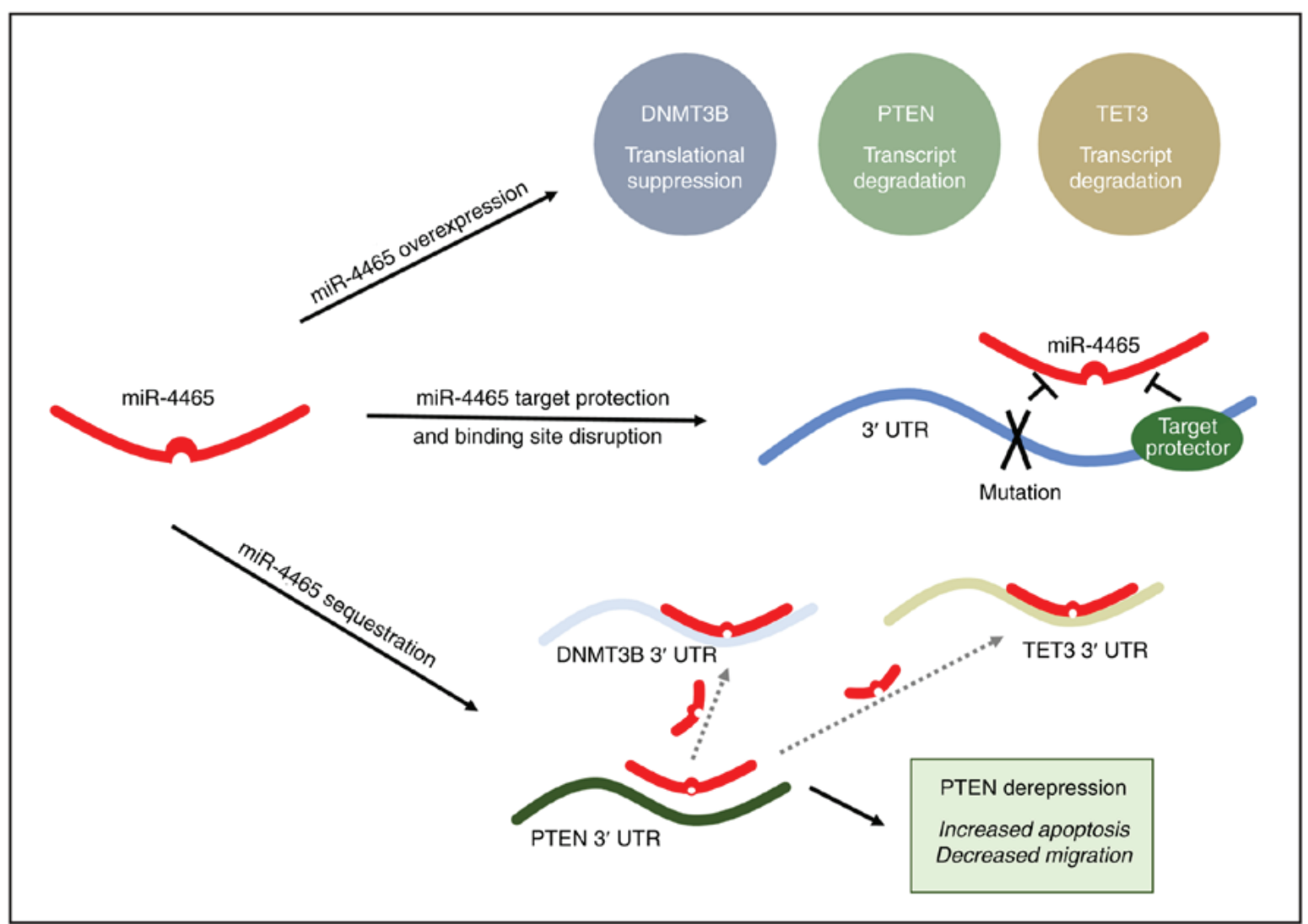

Figure 7. Graphical representation of the experimental approaches used to validate DNMT3B and TET3 as novel ceRNAs of the tumor suppressor PTEN, and miR-4465 as a shared miRNA among the three ceRNAs. DNMT3B, DNA methyltransferase 3 $\beta$; TET3, TET methylcytosine dioxygenase 3; ceRNA, competing endogenous RNA; miR, miRNA, microRNA; UTR, untranslated region.

conditions (Fig. 6D-F) compared with the siNEG controls. This may be the result of the abrogation of TET3 protein function as well as the redirection of shared miRNAs to other targets such as PTEN, as demonstrated in Fig. 4B. DNMT3B knockdown, compared with the siNEG control, exhibited pro-apoptotic and antimigratory effects in low-serum conditions (Fig. 6D-F) and an absence of an apparent effect in full-serum conditions (Fig. 6A-C), which may have overridden the effects of knockdown owing to full mitogenic stimulation. While this phenotypic readout may be attributed to the abrogation of the DNMT3B protein function as an oncogene, the results of DNMT3B 3'UTR overexpression (Figs. 4D and 5A-F) suggested a contribution of the miRNA-dependent, coding-independent function of DNMT3B.

\section{Discussion}

Multiple regulatory mechanisms responsible for downregulation of PTEN in cancer have been elucidated in the last two decades, including negative regulation by transcription factors, promoter hypermethylation, histone modifications, post-translational modifications, antisense RNA and miRNA regulation (24-29). Each one of these can have an impact on PTEN function by affecting its abundance, catalytic activity, protein interactions or localization (17-19). More recently, ceRNAs have been added to this growing list of PTEN regulators $(19,30)$. By virtue of shared miRNA binding sites, endogenous RNAs (mRNAs, lncRNAs and pseudogenes functioning as molecular decoys) can sponge up miRNAs away from their primary targets $(31,32)$.

The in silico identification and experimental validation of ceRNA networks in cancer are important for several reasons: i) It has helped establish the role of miRNAs in the regulation of oncogenes and tumor suppressors $(16,31,33,34)$; ii) it has revealed a novel, coding-independent and miRNA-dependent function for endogenous mRNAs $(16,19,30,31,35,36)$; iii) it has assigned a molecular decoy function to pseudogenes based on the extensive homology to and shared MREs with the parental gene $(31,37,38)$; iv) it has attributed another function to 3'UTRs of mRNA targets as trans regulatory elements that can modulate the expression of other RNAs through the sequestration of shared miRNAs (39); v) it has led to the identification of ceRNA hubs and higher-order ceRNA networks, as well as cancer-specific ceRNA modules (40-43).

The present study reported the experimental validation of two novel PTEN ceRNAs, DNMT3B and TET3, which were identified through the MREs that they share with PTEN in their 3'UTR regions. All three transcripts have previously been demonstrated to be subject to miRNA regulation. PTEN is regulated by its pseudogene molecular decoy, PTENP1, via a ceRNA network in colorectal and prostate cancer (31). In the present study, DNMT3B and TET3 were identified as additional ceRNAs that may positively modulate PTEN expression and enhance its tumor-suppressive functions in HCT116 colorectal cancer cells. This is, to the best of our knowledge, the first study to implicate both DNMT3B and 
TET3 in a ceRNA network. Previously, DNMT3B was only identified to be endogenously regulated by miRNAs in breast and liver cancer $(44,45)$. TET3 has only been demonstrated to be endogenously regulated by a network of miRNAs in the brain governing epigenetic mechanisms for memory formation and consolidation (46).

Evaluation of DNMT3B and TET3 as PTEN ceRNAs involved a series of experiments demonstrating that these putative ceRNAs may be regulated by shared miRNAs. miR-4465 was revealed to directly target and regulate all three transcripts via their 3'UTRs through a combination of luciferase reporter assays, abrogation of miRNA binding sites, target protection with locked nucleic acids and RT-qPCR assays. miRNA sequestration experiments including overexpression of individual 3'UTR fragments led to derepression of the ceRNAs. By contrast, knockdown of one of the ceRNAs through RNA interference led to decreased expression of the other two ceRNAs. These results suggested that indeed, PTEN, TET3 and DNMT3B may constitute a robust ceRNA network. Of note, although overexpression of 3'UTRs harboring miR-4465 binding sites may be largely responsible for miRNA sequestration in the 3'UTR overexpression experiments, knockdown by RNA interference affects the entire length of the transcript, which harbors multiple MREs targeted by multiple miRNAs. Thus, multiple shared miRNAs can be redirected and sequestered by the ceRNAs.

The effects of sequestration experiments on two cancer hallmarks, resistance to apoptosis and migratory capacity, revealed nuances of the ceRNA crosstalk. Overexpression of DNMT3B or TET3 3'UTR promoted apoptosis and decreased the migratory capacity potentially, at least in part, due to the shared miRNA sequestration and the consequent derepression of PTEN. In a physiological context, these may represent cellular conditions where either DNMT3B or TET3 is strongly upregulated, thus providing extra copies of the 3'UTR to sponge up miRNAs shared with PTEN. Fig. 7 summarizes the experimental approaches used to validate DNMT3B and TET3 as novel ceRNAs of PTEN.

Knockdown of TET3 resulted in an increased migratory capacity and resistance to apoptosis. The relative contribution of shared miRNAs redirected to PTEN to this phenotypic observation cannot easily be determined. Reduced expression of the TET3 protein, a known tumor suppressor, may have contributed to this result. Knockdown of DNMT3B did not exhibit phenotypes indicative of miRNA sequestration and instead promoted apoptosis and decreased the migratory capacity. This may mean that in this context, downregulation of DNMT3B in HCT116 colorectal cancer cells is phenotypically inconsequential. With fewer predicted shared MREs between PTEN and DNMT3B, the extra pool of shared miRNA made available by DNMT3B knockdown may not be enough to reverse the tumor-suppressive pro-apoptotic and anti-migratory phenotypic readout of PTEN expression. In addition, DNMT3B may participate in other ceRNA networks, and its knockdown may dissipate its miRNA regulators to various other target transcripts. The contribution of the coding-dependent function of DNMT3B, however, cannot be discounted; compared with a competitive miRNA sequestration experiment through 3'UTR overexpression, knockdown of DNMT3B may have simply decoupled its coding-dependent function as an oncogene from its miRNA-dependent, coding-independent function. TET3 knockdown, on the other hand, may elicit a pro-oncogenic phenotype by redirecting more shared miRNAs to its ceRNA PTEN, with which it shares 32 miRNA binding sites, compared with only 11 between PTEN and DNMT3B. However, similar to the case of DNMT3B knockdown, TET3 knockdown may have deprived the cells of a potent tumor suppressor protein. This highlights the disadvantages of the knockdown approach in studying potential ceRNAs; sequestration experiments using individual 3'UTR overexpression are more instructive. The value of using knockdown approaches lies in decoupling the protein-coding from the coding-independent function of mRNAs. In addition, these experiments highlight that functional relevance of ceRNA networks is determined largely by the number of shared miRNAs between or among ceRNAs, their relative stoichiometry and the relative affinity of shared miRNAs with the individual targets. Depending on these factors, redirection of shared miRNAs to competitors may not be relevant, as demonstrated by the dose-dependent tumor suppressive function of PTEN in the present study. Gene regulation in cancer is very dynamic, depending on cellular conditions at any point in time; there may or may not be specific contexts in which ceRNAs are expected to alter phenotypic outcomes.

In future studies, transfection experiments involving DNMT3B and TET3 cDNA, with or without their 3'UTRs, may help distinguish the coding-dependent and coding-independent functions of these genes. Full-length DNMT3B and TET3 overexpression experiments, however, come with at least one caveat. Considering the universal roles of the two genes in methylation and demethylation, respectively, of countless oncogenes and tumor suppressors, overexpression of their coding regions may not afford clear-cut interpretation of results.

The existence of a ceRNA network among PTEN, DNMT3B and TET3 may have important ramifications and merits further investigation. With the opposing functions of DNMT3B and TET3 as a de novo methylase and demethylase, respectively, the net effect of miRNA-dependent competition between DNMT3B and TET3, and among DNMT3B, TET3 and PTEN, may influence the methylation status of the PTEN promoter, and consequently, carcinogenesis.

\section{Acknowledgements}

The authors would like to thank Ms. Marie Isabelle Viola (Disease Molecular Biology and Epigenetics Laboratory, National Institute of Molecular Biology and Biotechnology, University of the Philippines Diliman) for assistance in the preparation of figures and statistical analyses and Mr. Charles Christopher Bataclan (Disease Molecular Biology and Epigenetics Laboratory, National Institute of Molecular Biology and Biotechnology, University of the Philippines Diliman) for sorting the references.

\section{Funding}

This work was supported by in-house funds from the National Institute of Molecular Biology and Biotechnology, University of the Philippines Diliman. 


\section{Availability of data and materials}

The datasets used and analyzed during the current study are available from the corresponding author on reasonable request.

\section{Authors' contributions}

RLG conceived the project and wrote the project proposal. KARR and KMMA performed the experiments. RLG, KMMA and KARR analyzed the data. RLG and KARR wrote the manuscript. All authors read and approved the final manuscript.

\section{Ethics approval and consent to participate}

Not applicable.

\section{Patient consent for publication}

Not applicable.

\section{Competing interests}

The authors declare that they have no competing interests.

\section{References}

1. Carnero A, Blanco-Aparicio C, Renner O, Link W and Leal J: The PTEN/PI3K/AKT signalling pathway in cancer, therapeutic implications. Curr Cancer Drug Targets 8: 187-198, 2008.

2. Sun Z, Huang C, He J, Lamb KL, Kang X, Gu T, Shen WH and Yin Y: PTEN C-terminal deletion causes genomic instability and tumor development. Cell Rep 6: 844-854, 2014.

3. Murphy SJ, Karnes RJ, Kosari F, Castellar BE, Kipp BR, Johnson SH, Terra S, Harris FR, Halling GC, Klein JL, et al Integrated analysis of the genomic instability of PTEN in clinically insignificant and significant prostate cancer. Mod Pathol 29: 143-156, 2016.

4. Molinari F and Frattini M: Functions and regulation of the PTEN gene in colorectal cancer. Front Oncol 3: 326, 2014.

5. Berger AH, Knudson AG and Pandolfi PP: A continuum model for tumour suppression. Nature 476: 163-169, 2011.

6. Alimonti A, Carracedo A, Clohessy JG, Trotman LC, Nardella C Egia A, Salmena L, Sampieri K, Haveman WJ, Brogi E, et al: Subtle variations in Pten dose determine cancer susceptibility. Nat Genet 42: 454-458, 2010.

7. Trotman LC, Niki M, Dotan ZA, Koutcher JA, Di Cristofano A, Xiao A, Khoo AS, Roy-Burman P, Greenberg NM, Van Dyke T, et al: Pten dose dictates cancer progression in the prostate. PLoS Biol 1: E59, 2003.

8. Garofalo M,Di Leva G, Romano G, Nuovo G, Suh SS, Ngankeu A, Taccioli C, Pichiorri F, Alder H, Secchiero P, et al: miR-221\&222 regulate TRAIL resistance and enhance tumorigenicity through PTEN and TIMP3 downregulation. Cancer Cell 16: 498-509, 2009.

9. Huse JT, Brennan C, Hambardzumyan D, Wee B, Pena J, Rouhanifard SH, Sohn-Lee C, le Sage C, Agami R, Tuschl T and Holland EC: The PTEN-regulating microRNA miR-26a is amplified in high-grade glioma and facilitates gliomagenesis in vivo. Genes Dev 23: 1327-1337, 2009.

10. Tabach Y, Billi AC, Hayes GD, Newman MA, Zuk O, Gabel H, Kamath R, Yacoby K, Chapman B, Garcia SM, et al: Identification of small RNA pathway genes using patterns of phylogenetic conservation and divergence. Nature 493: 694-698, 2013.

11. Mullany LE, Herrick JS, Wolff RK, Stevens JR, Samowitz W and Slattery ML: MicroRNA-transcription factor interactions and their combined effect on target gene expression in colon cancer cases. Genes, Chromosom Cancer 57: 192-202, 2018.
12. Volinia S, Calin GA, Liu CG, Ambs S, Cimmino A, Petrocca F, Visone R, Iorio M, Roldo C, Ferracin M, et al: A microRNA expression signature of human solid tumors defines cancer gene targets. Proc Natl Acad Sci USA 103: 2257-2261, 2006.

13. Frankel LB, Christoffersen NR, Jacobsen A, Lindow M, Krogh A and Lund AH: Programmed cell death 4 (PDCD4) is an important functional target of the microRNA miR-21 in breast cancer cells. J Biol Chem 283: 1026-1033, 2008.

14. Zhu S, Si ML, Wu H and Mo YY: MicroRNA-21 targets the tumor suppressor gene tropomyosin 1 (TPM1). J Biol Chem 282 14328-14336, 2007.

15. Chan JA, Krichevsky AM and Kosik KS: MicroRNA-21 Is an antiapoptotic factor in human glioblastoma cells. Cancer Res 65: 6029-6033, 2005.

16. Tay Y, Rinn J and Pandolfi PP: The multilayered complexity of ceRNA crosstalk and competition. Nature 505: 344-352, 2014.

17. Zarringhalam K, Tay Y, Kulkarni P, Bester AC, Pandolfi PP and Kulkarni RV: Identification of competing endogenous RNAs of the tumor suppressor gene PTEN: A probabilistic approach. Sci Rep 7: 7755, 2017.

18. Poliseno L and Pandolfi PP: PTEN ceRNA networks in human cancer. Methods 77-78: 41-50, 2015.

19. Karreth FA, Tay Y, Perna D, Ala U, Tan SM, Rust AG, DeNicola G, Webster KA, Weiss D, Perez-Mancera PA, et al: In vivo identification of tumor-suppressive PTEN ceRNAs in an oncogenic BRAF-induced mouse model of melanoma. Cell 147: 382-395, 2011.

20. Marone M, Mozzetti S, De Ritis D, Pierelli L and Scambia G: Semiquantitative RT-PCR analysis to assess the expression levels of multiple transcripts from the same sample. Biol Proced Online 3: 19-25, 2001

21. Langlois MJ, Bergeron S, Bernatchez G, Boudreau F, Saucier C, Perreault N, Carrier JC and Rivard N: The PTEN phosphatase controls intestinal epithelial cell polarity and barrier function: Role in colorectal cancer progression. PLoS One 5: e15742, 2010.

22. Rhee I, Bachman KE, Park BH, Jair KW, Yen RW, Schuebel KE, Cui H, Feinberg AP, Lengauer C, Kinzler KW, et al: DNMT1 and DNMT3b cooperate to silence genes in human cancer cells. Nature 416: 552-556, 2002.

23. Uribe-Lewis S, Stark R, Carroll T, Dunning MJ, Bachman M, Ito Y, Stojic L, Halim S, Vowler SL, Lynch AG, et al: 5-hydroxymethylcytosine marks promoters in colon that resist DNA hypermethylation in cancer. Genome Biol 16: 69, 2015.

24. García JM, Silva J, Peña C, Garcia V, Rodríguez R, Cruz MA, Cantos B, Provencio M, España P and Bonilla F: Promoter methylation of the PTEN gene is a common molecular change in breast cancer. Genes Chromosom Cancer 41: 117-124, 2004.

25. Alvarez-Nuñez F, Bussaglia E, Mauricio D, Ybarra J, Vilar M, Lerma E, de Leiva A and Matias-Guiu X; Thyroid Neoplasia Study Group: PTEN promoter methylation in sporadic thyroid carcinomas. Thyroid 16: 17-23, 2006.

26. Lu J, Jeong H, Kong N, Yang Y, Carroll J, Luo HR, Silberstein LE, Yupoma and Chai L: Stem cell factor SALL4 represses the transcriptions of PTEN and SALL1 through an epigenetic repressor complex. PLoS One 4: e5577, 2009.

27. Hettinger K, Vikhanskaya F, Poh MK, Lee MK, de Belle I, Zhang JT, Reddy SA and Sabapathy K: c-Jun promotes cellular survival by suppression of PTEN. Cell Death Differ 14: 218-229, 2007.

28. Vasudevan KM, Gurumurthy S and Rangnekar VM: Suppression of PTEN expression by NF-kappa B prevents apoptosis. Mol Cell Biol 24: 1007-1021, 2004.

29. Meng F, Henson R, Wehbe-Janek H, Ghoshal K, Jacob ST and Patel T: MicroRNA-21 regulates expression of the PTEN tumor suppressor gene in human hepatocellular cancer. Gastroenterology 133: 647-658, 2007.

30. Tay Y, Kats L, Salmena L, Weiss D, Tan SM, Ala U, Karreth F, Poliseno L, Provero P, Di Cunto F, et al: Coding-independent regulation of the tumor suppressor PTEN by competing endogenous mRNAs. Cell 147: 344-357, 2011.

31. Poliseno L, Salmena L, Zhang J, Carver B, Haveman WJ and Pandolfi PP: A coding-independent function of gene and pseudogene mRNAs regulates tumour biology. Nature 465: 1033-1038, 2010.

32. Salmena L, Poliseno L, Tay Y, Kats L and Pandolfi PP: A ceRNA Hypothesis: The rosetta stone of a hidden RNA language? Cell 146: 353-358, 2011.

33. Yamakuchi M, Lotterman CD, Bao C, Hruban RH, Karim B, Mendell JT, Huso D and Lowenstein CJ: P53-induced microRNA-107 inhibits HIF-1 and tumor angiogenesis. Proc Natl Acad Sci USA 107: 6334-6339, 2010. 
34. Wang B, Hsu SH, Wang X, Kutay H, Bid HK, Yu J, Ganju RK, Jacob ST, Yuneva $M$ and Ghoshal K: Reciprocal regulation of microRNA-122 and c-Myc in hepatocellular cancer: Role of E2F1 and transcription factor dimerization partner 2. Hepatology 59: 555-566, 2014.

35. Sumazin P, Yang X, Chiu HS, Chung WJ, Iyer A, Llobet-Navas D, Rajbhandari P, Bansal M, Guarnieri P, Silva J and Califano A: An Extensive MicroRNA-mediated network of RNA-RNA interactions regulates established oncogenic pathways in glioblastoma. Cell 147: 370-381, 2011.

36. Jeyapalan Z, Deng Z, Shatseva T, Fang L, He C and Yang BB: Expression of $\mathrm{CD} 443$ '-untranslated region regulates endogenous microRNA functions in tumorigenesis and angiogenesis. Nucleic Acids Res 39: 3026-3041, 2011.

37. Rutnam ZJ, Du WW, Yang W, Yang X and Yang BB: The pseudogene TUSC2P promotes TUSC2 function by binding multiple microRNAs. Nat Commun 5: 2914, 2014.

38. Karreth FA, Reschke M, Ruocco A, Ng C, Chapuy B, Léopold V, Sjoberg M, Keane TM, Verma A, Ala U, et al: The BRAF pseudogene functions as a competitive endogenous RNA and induces lymphoma in vivo. Cell 161: 319-332, 2015

39. Wang L, Guo ZY, Zhang R, Xin B, Chen R, Zhao J, Wang T, Wen WH, Jia LT, Yao LB and Yang AG: Pseudogene OCT4-pg4 functions as a natural micro RNA sponge to regulate OCT4 expression by competing for miR-145 in hepatocellular carcinoma. Carcinogenesis 34: 1773-1781, 2013.

40. Sanchez-Mejias A and Tay Y: Competing endogenous RNA networks: Tying the essential knots for cancer biology and therapeutics. J Hematol Oncol 8: 30, 2015.

41. Ergun S and Oztuzcu S: Oncocers: ceRNA-mediated cross-talk by sponging miRNAs in oncogenic pathways. Tumor Biol 36: 3129-3136, 2015.
42. Park SM, Gaur AB, Lengyel E and Peter ME: The miR-200 family determines the epithelial phenotype of cancer cells by targeting the E-cadherin repressors ZEB1 and ZEB2. Genes Dev 22: 894-907, 2008.

43. Xu J, Li Y, Lu J, Pan T, Ding N, Wang Z, Shao T, Zhang J, Wang $\mathrm{L}$ and $\mathrm{Li} \mathrm{X}$ : The mRNA related ceRNA-ceRNA landscape and significance across 20 major cancer types. Nucleic Acids Res 43: 8169-8182, 2015.

44. Chen L, Zheng J, Zhang Y, Yang L, Wang J, Ni J, Cui D, Yu C and Cai Z: Tumor-specific Expression of MicroRNA-26a suppresses human hepatocellular carcinoma growth via cyclin-dependent and -independent pathways. Mol Ther 19: 1521-1528, 2011.

45. Sandhu R, Rivenbark AG and Coleman WB: Enhancement of chemotherapeutic efficacy in hypermethylator breast cancer cells through targeted and pharmacologic inhibition of DNMT3b. Breast Cancer Res Treat 131: 385-399, 2012.

46. Noack F and Calegari F: MicroRNAs meet epigenetics to make for better brains. EMBO Rep 15: 1224-1225, 2014

47. Garcia DM, Baek D, Shin C, Bell GW, Grimson A and Bartel DP: Weak seed-pairing stability and high target-site abundance decrease the proficiency of lsy-6 and other miRNAs. Nat Struct Mol Biol 18: 1139-1146, 2011.

48. Friedman RC, Farh KK, Burge CB and Bartel DP: Most mammalian mRNAs are conserved targets of microRNAs. Genome Res 19: 92-105, 2009.

(i) (2) This work is licensed under a Creative Commons Attribution-NonCommercial-NoDerivatives 4.0 International (CC BY-NC-ND 4.0) License. 